\title{
Manipulating Characteristic Timescales and Fiber Morphology in Simultaneous Centrifugal Spinning and Photopolymerization
}

Yichen Fang ${ }^{1}$, Austin D. Dulaney ${ }^{1}$, Jesse Gadley ${ }^{2}$, Joao M. Maia ${ }^{2}$ and Christopher J. Ellison ${ }^{1, *}$

${ }^{1}$ McKetta Department of Chemical Engineering, The University of Texas at Austin, Austin, TX 78712, USA

${ }^{2}$ Department of Macromolecular Science and Engineering, Case Western Reserve University, Cleveland, OH USA

*To whom correspondence should be addressed: ellison@che.utexas.edu

\section{Abstract}

In this study, the fabrication of crosslinked nonwoven fibers via simultaneous thiol-ene photopolymerization and spinning of monomer jets has been demonstrated in centrifugal Forcespinning for the first time. We observed that simultaneous Forcespinning and photopolymerization resulted in a wide variety of fiber morphologies including beads, beadson-string, uniform fiber, fused fibers, and well-cured fibers. To elucidate the underlying mechanisms and parameter interactions that give rise to these morphologies, we systematically varied the light intensity, solution elasticity, and spin speed of the Forcespinning process. From these experimental results, an operating diagram was constructed based on the measured process parameters, their respective timescales, and observed effects on fiber morphology. While numerous parameters can individually affect fiber formation and morphology, the interplay between curing kinetics, solution viscoelasticity, and orifice-to-collector processing time window is also crucial in this process. Smooth and well-cured fibers were formed only when the photopolymerization occurred sufficiently quickly, before both the breakup of fibers 
into droplets due to a surface tension driven Rayleigh instability and the deposition of fibers on the collector. Our findings can serve as a predictive guideline for the fabrication of crosslinked fibers with desired morphology, the implementation of the in-situ polymerization and spinning concept into other commercial fiber manufacturing technologies, and the adaptation of other functional or high performance monomer systems.

\section{Introduction}

Nowovens are composed of randomly oriented fibers held together by physical entanglements, or bonded by chemical, mechanical or thermal. They possess many unique properties such as high surface area to volume ratio and high surface functionality, usually processed into the form of a lightweight, porous mat. Recent advances in fiber production technologies have led to increasing opportunities to utilize nonwoven fibers for diverse applications such as filtration [1, 2], superhydrophobic materials [3-5], catalysis [6], and tissue engineering [7-9], among others.

Currently, most nonwoven fibers with submicron or single micron average diameters are produced by melt blowing, electrospinning, or adaptations of the two, such as solution blow spinning [10] or melt state electrospinning [11, 12]. In melt blowing, molten polymer is extruded through a die and drawn into thin continuous fibers by high velocity hot air jets. The liquid fiber jet solidifies when the processing temperature drops below the glass transition or crystallization temperature of the polymer. Alternatively, for electrospinning a polymeric solution is delivered through a nozzle by a syringe pump and elongated into a jet under the application of a strong electric field. The fiber jet elongates and solidifies into fine fibers as the solvent rapidly evaporates. In recent years, another fiber production technology, termed 
centrifugal spinning or Forcespinning, has attracted a significant amount of interest [13]. In this process, a polymer melt or solution is delivered through the orifices of a fast rotating spinneret. When the centrifugal force and the hydrostatic pressure overcome the surface tension of the drop formed at the orifice outlet, the droplet is stretched into a fluid jet and attenuated, often into submicron fibers. Forcespinning has been shown to be a versatile and high throughput method with the ability to process a variety of polymeric and inorganic materials in the melt or solution state [14-17].

All of the current fiber production technologies fundamentally involve transforming a preformed polymer into a liquid by either the application of heat or the addition of solvent, and subsequently applying an extensional drawing force to draw the liquid into fibers, which later solidify by sufficient cooling or solvent evaporation. Processing with solvents or heat has both advantages and drawbacks. Melt state processing, although simple and convenient, requires significant thermal energy input [18] and has its upper processing limit constrained by the degradation temperature of the material. On the other hand, solution state processing is quite versatile and can be performed at room temperature but suffers from issues such as solvent recovery/recycling, safety of volatile/flammable solvents, and low process throughput, all which present challenges for scale up and commercial viability.

Recently, our group demonstrated a new method for fabricating nonwoven fibers directly from non-volatile liquid monomer mixtures instead of from polymer solutions or polymer melts [19], thereby foregoing the use of solvent and heat altogether. In this method, a mixture containing monomers and a photoinitiator was simultaneously electrospun and photopolymerized via UV initiated thiol-ene chemistry to produce solid fibers in ambient 
conditions. The method was also made even greener by replacing more than $50 \mathrm{wt} \%$ of petroleum based monomer with a bio-renewable soybean oil derived monomer without sacrificing the final fiber quality [20]. Despite the very limited curing time during the fiber spinning process, the fibers made by this method contained a highly crosslinked network, which imparted high thermal stability, chemical resistance, and mechanical strength to the resulting fibers [19]. Owing to the versatility of thiol-ene chemistry, fibers with drastically different mechanical, thermal, and network structural properties were fabricated by incorporating monomers with different chemical structures [21].

Other researchers have also reported related strategies for in-situ photocrosslinking during fiber formation. Notably, Boyd and coworkers successfully fabricated thiol-ene and thiol-yne fibers using hydrodynamic shaping in microfluidic channels [22]. In other studies, an extra step of thermal polymerization to convert some amount of the monomers into prepolymers prior to electrospinning or the incorporation of a high molecular weight (MW) polymer in significant amounts was necessary to produce crosslinked fibers due to the slow reaction kinetics [23-25]. However, most studies only report the chemical formulations and processing conditions that generated fibers, but do not address the many other factors that impact fiber formation and fiber morphology. In addition, the reported conditions usually do not reflect the entire operating space or the extent of tunability of the methods.

In contrast, controlling fiber formation and morphology has long been the subject of research for the more commonly studied fiber spinning techniques, since fiber diameter, quality and morphology ultimately determine the properties $[1,3,4]$, and in turn, the end use of the fibers. For example, the effect of solvent properties, including solvent quality, 
conductivity, volatility, and surface tension, on generating fused, beaded, and uniform electrospun fibers has been investigated in great detail [26-28]. Other studies have examined the lower polymer concentration limit for fiber formation in electrospinning. They correlated the formation of beads, beaded fibers, bead-free and uniform fibers with the degree of chain entanglements in the polymer solution [29-33], solution elasticity [34], molecular weight distribution [34, 35], and polymer architecture (branched polymer versus linear polymer) [31].

Although the simultaneous photopolymerization and fiber spinning process described herein may appear similar to electrospinning in that they are both solution-based processes, there are several key differences. First, the solutions for simultaneous photopolymerization and fiber spinning consist mainly of monomers that are essentially non-volatile, unlike the polymeric solutions used in electrospinning, which often contain large amounts of volatile solvent. Naturally, these two different solutions are expected to have different viscoelastic properties, which will greatly influence the extensional behavior of the fiber jets during fiber spinning and in turn the morphological appearance of fibers. Second, the fiber jets herein solidify due to rapid photopolymerization triggered by UV light, instead of rapid solvent evaporation as for traditional electrospinning. The incorporation of in-situ photopolymerization is a unique feature to fiber spinning, and therefore the role and importance of reaction kinetics on fiber formation and morphology has become a new area for fundamental study. To date, a systematic and fundamental examination of the important parameters and underlying physics that govern fiber formation and fiber morphology in simultaneous photopolymerization and fiber spinning is still lacking and this motivates the present study. 
Here, we demonstrate for the first time the integration of thiol-ene photopolymerization with a bench-top centrifugal Forcespinning apparatus equipped with a custom UV light source. The successful implementation demonstrates not only the universality (previous studies employed an electrospinning apparatus without using solvent) but also the scale-up potential of this concept, as Forcespinning's intrinsically larger fiber drawing force and higher throughput constitute significant advantages over electrospinning. In this process, the principle parameters that govern fiber formation and morphology include: reaction parameters -stoichiometric ratio of thiol to ene, monomer functionality and reactivity, light intensity, and photoinitiator content; viscoelastic parameters - viscosity and elasticity; and process parameters - spin speed, orifice diameter, and the location and width of the UV illuminated region. These three categories of parameters form a convoluted and in some cases interactive set of variables, making it challenging to study the effect of each variable in isolation. For example, a penta-functional acrylate monomer is different from a tetra-functional acrylate in terms of both monomer functionality and viscosity.

To overcome this complication, we characterized the timescales related to these categories: namely those of the reaction kinetics, viscoelastic response, and the orifice-tocollector processing time. By carefully selecting variables that are associated with only one of the aforementioned timescales, the morphology of the photopolymerized fibers could be finely tuned while keeping all other parameters constant. In this study, the light intensity, the concentration of an elasticity modifier, and the spin speed of the spinneret were varied systematically to change the reaction kinetics, the viscoelastic response, and the processing time, respectively. The morphological appearances of the resulting fibers, ranging from beads, 
beads-on-string, uniform fibers, fused fibers, to well cured fibers, were characterized using scanning electron microscopy (SEM), while the timescales were measured experimentally by a variety of characterization methods as described in detail in the following sections.

Finally, the morphological appearance and their timescales were superimposed to construct an operating diagram for the photopolymerization Forcespinning process. It was found that uniform and well-cured fibers were produced only when the three timescales were closely matched; fiber defects started to develop as one or more timescales deviated from the optimal values. These experiments and the resulting operating diagram elucidated the role of each timescale as well as the intricate interplay between the timescales and their effects on the formation and the morphological evolution of the fibers. With this fundamental knowledge, the process could be readily adapted to include many different monomer chemistries to produce a diverse profile of fibers, and implemented within other industrial fiber production technologies for widespread use in manufacturing.

\section{Experimental}

\subsection{Materials and solution preparation}

Dipentaerythritol pentaacrylate (5A) was provided by Sartomer USA. Pentaerythritol tetrakis (3-mercaptoproprionate) (4T) was purchased from Sigma Aldrich. A commercially available photoinitiator, Irgacure 2100 , was kindly provided by BASF Switzerland. Polyethylene oxide (PEO), with a viscosity average molecular weight (MW) of $10^{6} \mathrm{~g} / \mathrm{mol}$, and anhydrous ethyl acetate were obtained from Sigma Aldrich. All chemicals were used as received. 
To impart elasticity to the monomer mix solutions and prevent fiber jet breakup, a trace amount of PEO was included in the monomer mix formulation. Because PEO did not readily dissolve in the monomer mix solution at room temperature, the appropriate amount of PEO was first added to $4 \mathrm{~T}$ at an elevated temperature. PEO containing $4 \mathrm{~T}$ was heated at $70{ }^{\circ} \mathrm{C}$ for 18 hours to ensure complete dissolution. It is worth noting that prolonged heating of PEO/4T solution should be avoided, since oxidative degradation of PEO becomes significant at temperatures above the melting point of PEO, which results in reduced PEO MW [36] and reduced elasticity of the PEO containing solution.

The monomer mix solution was prepared by mixing together 66 wt\% 5A, 19 wt\% 4T containing PEO, $3 \mathrm{wt} \%$ Irgacure 2100, and $12 \mathrm{wt} \%$ ethyl acetate using a vortex mixer (Thermolyne 37600). This solution composition yielded an ene to thiol functional group molar ratio of 4 to 1 . This stoichiometric ratio has been shown suitable for fiber spinning processes by providing both sufficient photopolymerization kinetics and a reduced oxygen inhibition effect due to the presence of thiol [19-21]. In addition, ethyl acetate was added as a diluent to reduce the viscosity of the monomer mix solution, which ensures sufficient solution delivery through the spinneret orifices. Ethyl acetate can also be replaced by reactive monomers with low viscosity such as 1,6-hexanediol diacrylate, which can be polymerized into the network. However, ethyl acetate was a preferred diluent in this particular study as it reduces the solution viscosity without affecting the photopolymerization kinetics. The monomer mix solution was prepared in a room with no stray UV light, to prevent pre-exposure that could initiate the photopolymerization prior to fiber spinning.

\subsection{Light design and centrifugal Forcespinning}


A bench scale centrifugal Forcespinning apparatus (FibeRio Cyclone L-1000) was equipped with a custom made UV light source as shown in Fig. 1. The light source arrangement included a power supply (Photon Technology International LPS-220), a lamp housing (Photon Technology International A1010) for a mercury arc lamp (Ushio), and a UV transparent mirror (Edmund Optics) mounted at a $45{ }^{\circ} \mathrm{C}$ angle to reflect the light vertically onto the collector, with an illumination spot $10 \mathrm{~cm}$ in diameter. In this process, the monomer jets undergo simultaneous filament thinning and solidification through thiol-ene polymerization as they pass through this illuminated spot and deposit onto the vertical stainless steel collector bars.

For fiber spinning, $2 \mathrm{~mL}$ of monomer mix solution was loaded into the spinneret attached to two needle tips (inner diameter of 210 microns, 1.5 inch length). With this spinneret geometry, the distance from each orifice opening to the spinning axis was $11.2 \mathrm{~cm}$. The monomer mix solutions were spun at specified light exposure and spin speed conditions. Finally, scanning electron microscopy (SEM, Hitachi S-5500) was used to characterize the fiber diameter and morphology.
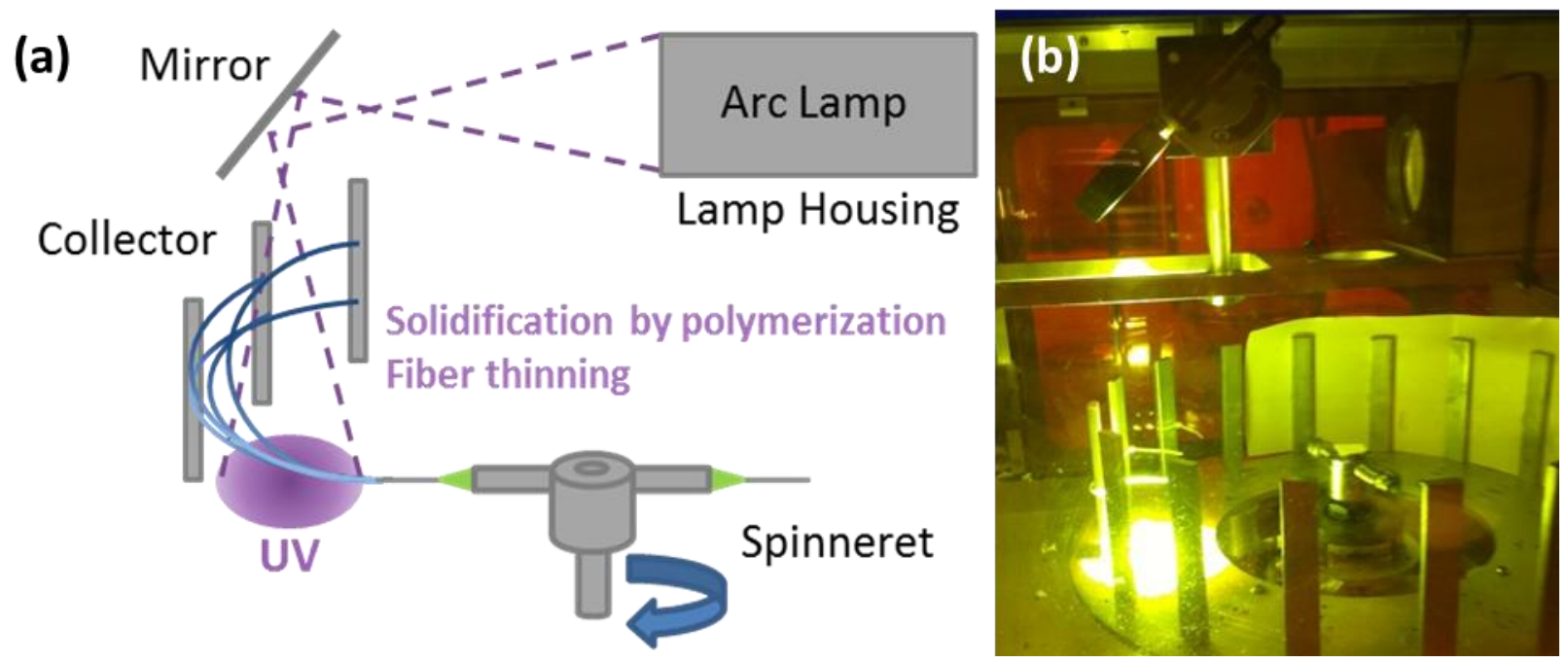
Fig. 1. (a) A schematic of the apparatus and (b) a photograph of the UV light design adopted in centrifugal Forcespinning.

\subsection{Real time Fourier transform infra-red spectroscopy (RT-FTIR)}

RT-FTIR (Thermo Scientific Nicolet 6700 FT-IR) was employed to determine the curing kinetics of the monomer mix solution exposed at different light intensities. A drop of freshly prepared monomer mix solution was sandwiched between two $\mathrm{NaCl}$ windows to form a continuous film. This sample preparation protocol prevented the evaporation of ethyl acetate and ensured the sample was thin enough for both UV (for initiating polymerization) and IR (for tracking polymerization kinetics) light to transmit through the film. A spot-cure xenon lamp (Optical Building Blocks Scopelite 200) with a collimating lens was fixed at a distance of $10 \mathrm{~cm}$ from the sample. To adjust the light intensity output, UV neutral density filters with optical densities of $0.3,0.6$, and 1.0 (Thorlabs) were used.

However, the xenon lamp, used in the RT-FTIR studies, and the mercury arc lamp, used in the fiber spinning runs, have different spectral output and light intensity. In order to accurately track the polymerization kinetics in the fiber spinning process, we define the effective light intensity, $l_{\text {eff, to }}$ be the light intensity at wavelengths lower than $435 \mathrm{~nm}$, which is the absorbance range of the photoinitiator used in this study. Here we assume that only the light with such wavelengths can effectively excite the photoinitiator to generate radicals and that the light excitation efficiency in this wavelength range is $100 \%$. A $435 \mathrm{~nm}$ band pass filter (Thor lab) was used to measure the light intensity at wavelengths of $435 \mathrm{~nm}$ and above, which was then subtracted from the light intensity at all wavelengths to calculate $l_{\text {eff. }}$ The absolute light 
intensity and $\mathrm{l}_{\text {eff }}$ of the light sources used in RT-FTIR and fiber spinning studies were measured by a radiometer (Coherent Field Max II). The measurements and the light intensity calculations are summarized in Table S1 - S3 in Supporting Information. UV-vis spectra of filters used in these studies are shown in Figure S1.

\subsection{Capillary breakup extensional rheometer (CaBER) measurements}

The extensional properties of the monomer mix solutions containing various amounts of PEO, ranging from $0 \mathrm{ppm}$ to $600 \mathrm{ppm}$, were measured by CaBER (Thermo Haake CaBER 1) equipped with $4 \mathrm{~mm}$ diameter plates. The filament diameter evolution with time was recorded by a laser micrometer at $1000 \mathrm{~Hz}$. Five measurements were collected for each solution.

The density of the monomer mix solution was determined to be $1.15 \mathrm{~g} / \mathrm{cm}^{3}$ by measuring the weight of a known volume of the solution. The surface tension was determined using an axisymmetric pendant droplet of the monomer mix with methods described previously [37]. The droplet was held for 2 minutes to equilibrate before measurements were made. The droplet shape profile was fitted according to the Young/Laplace equation with a software package (CAM200, KSV Ltd., Finland). The surface tension of the monomer mix solution was measured to be $40.93 \mathrm{mN} / \mathrm{m}$.

The solutions used in the CaBER analysis as well as the density and surface tension characterizations contained no photoinitiator to simplify the measurements, as we assumed that such low loadings of photoinitiator in our studies did not alter the viscoelastic and physical properties of the solution significantly.

\section{Results and discussion}




\subsection{Fiber morphology evolved with light intensity and characterization of gel time}

In thiol-ene chemistry, the reaction kinetics can be manipulated in a number of ways, for instance, by changing the functionality of monomers, changing the stoichiometric ratio of ene to thiol functional groups, or using other ene functional groups with different reactivity. However, varying these parameters can also lead to changes in the viscoelasticity of the monomer mix and types of polymerization (chain growth, step growth, etc.) that occur. In order to isolate a single independent variable, we chose instead to vary $l_{\text {eff, }}$ which allowed tuning of the reaction kinetics while keeping solution properties and other processing parameters constant.

A series of 5A-4T monomer mix solutions with optimal composition: $0.02 \mathrm{wt} \%$ PEO, $3 \mathrm{wt} \%$ Irgacure 2100 , and 12 wt\% ethyl acetate, were processed at $2500 \mathrm{rpm}$ and at various $\mathrm{I}_{\text {eff }}$ ranging from 629 to $24 \mathrm{~mW} / \mathrm{cm}^{2}$ by changing the voltage supplied to the light source. The resulting fibers are shown in Fig. 2. At high $\mathrm{l}_{\mathrm{eff}}$ (Fig. 2 a-c), the fibers are uniform in diameter and well cured. As $\mathrm{l}_{\text {eff }}$ incrementally decreases to $259 \mathrm{~mW} / \mathrm{cm}^{2}$ (Fig. 2 d), the fibers developed fused junctions, indicating that they are not fully cured when deposited onto the collector. As $I_{\text {eff }}$ continues to decrease (Fig. 2 e-h), the fibers also become increasingly less uniform in diameter. In this range of $\mathrm{I}_{\mathrm{eff}}$, the onset of surface tension driven Rayleigh instabilities occur prior to solidification. Rayleigh instabilities result in necking along the fibers axis and associated periodic variations in fiber diameter. When $l_{\text {eff }}$ drops below $21 \mathrm{~mW} / \mathrm{cm}^{2}$ (Fig. 2 i), the solidification via photopolymerization occurs so slowly that the instability has caused complete breakup of filaments, depositing only droplets on the collector. 

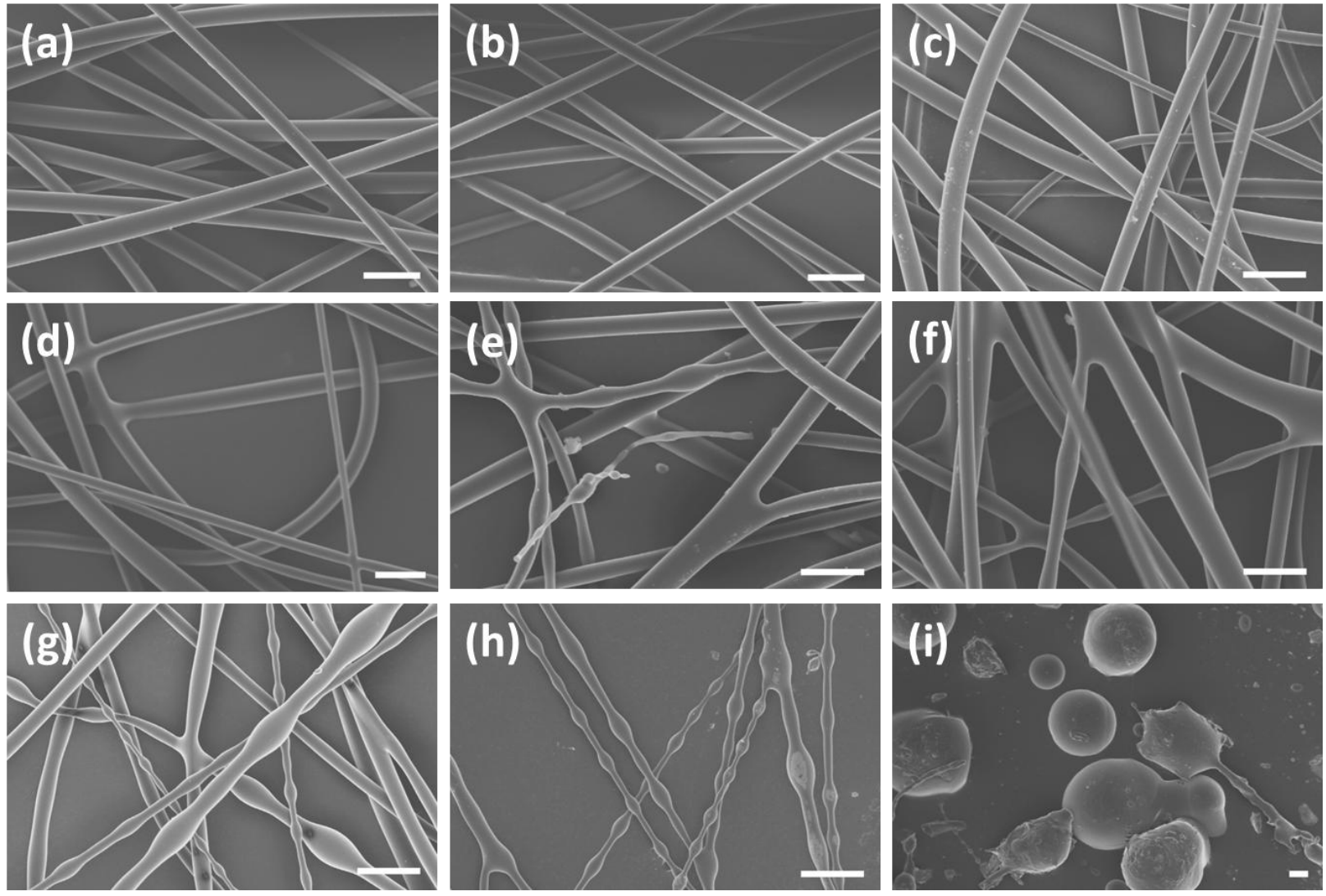

Fig. 2. SEM images of fibers Forcespun from monomer mix containing 200 ppm PEO, at a spin speed of $2500 \mathrm{rpm}$, and $\mathrm{I}_{\text {eff }}$ of (a) 629, (b) 571, (c) 403, (d) 305, (e) 259, (f) 195, (g) 115, (h) 38, and (i) $21 \mathrm{~mW} / \mathrm{cm}^{2}$, respectively. The scale bars in this figure corresponds to $25 \mu \mathrm{m}$.

To quantitatively investigate how the photopolymerization kinetics evolves with light intensity and how the kinetics timescale relates to the instability growth timescale and the fiber spinning timescale, RT-FTIR studies were performed on the aforementioned spinning mixture at various light intensities. A useful way to describe the curing kinetics of a cross-linked network is by its gel point, the point at which a continuous, cross-linked network is formed and the gel time $\left(T_{\text {gel }}\right)$, the time for a monomer mixture to reach the gel point. According to the gelation theory developed by Bowman and coworkers, the gel point of a mixed chain growth and step 
growth cross-linked network such as the one under investigation in this report can be calculated using the equation below [20, 38]:

$$
\frac{2}{r}\left(f_{\text {ene }}-1\right) \frac{k_{C C}}{k_{C S}} p_{\alpha}+\left(f_{\text {ene }}-1\right)\left(f_{S H}-1\right)\left(1+\frac{2}{r} \frac{k_{C C}}{k_{C S}}\right) p_{\alpha}^{2}=1
$$

where $r$ is the stoichiometric ratio of thiol to ene, $f_{\text {ene }}$ and $f_{S H}$ are the functionality of ene and thiol monomer, respectively, $\mathrm{p}_{\alpha}$ is the gel point in terms of degree of acrylate conversion, and $k_{c c} / k_{c s}$ is the ratio of the propagation constant for acrylate-acrylate reactions to the chain transfer constant of acrylate-thiol reactions, which has been previously reported to be around $1.5[20,38,39]$. The gel point was calculated to be $2.2 \%$ of the acrylate double bond conversion. The double bond conversion over time was tracked by the reduction of the peak area of the acrylate group at $1636 \mathrm{~cm}^{-1}[19,20,40]$.

Fig. 3 is a typical RT-FTIR kinetics curve of the monomer mix solution, with time zero being the onset of the UV exposure. The inset shows the double bond conversion at longer times. Because the gel time of the monomer mix solution occurs so fast that it nearly approaches the resolution of the instrument, directly interpolating gel time using a few data points does not yield very accurate or consistent results. Instead, the conversion-time data was fit up to $20 \%$ conversion, where polymerization rate is zeroth order with respect to acrylate double bonds [41], to a least squares fit linear equation as indicated by the solid line in Fig. 3. The gel time was then calculated by linear fit equation. The polymerization kinetics at five different light intensities were measured and data were processed as described. The gel times were calculated according to Eq. 1 as values averaged between four independent RT-FTIR measurements at each $\mathrm{l}_{\text {eff. }}$ The results are presented in Fig. 4. 


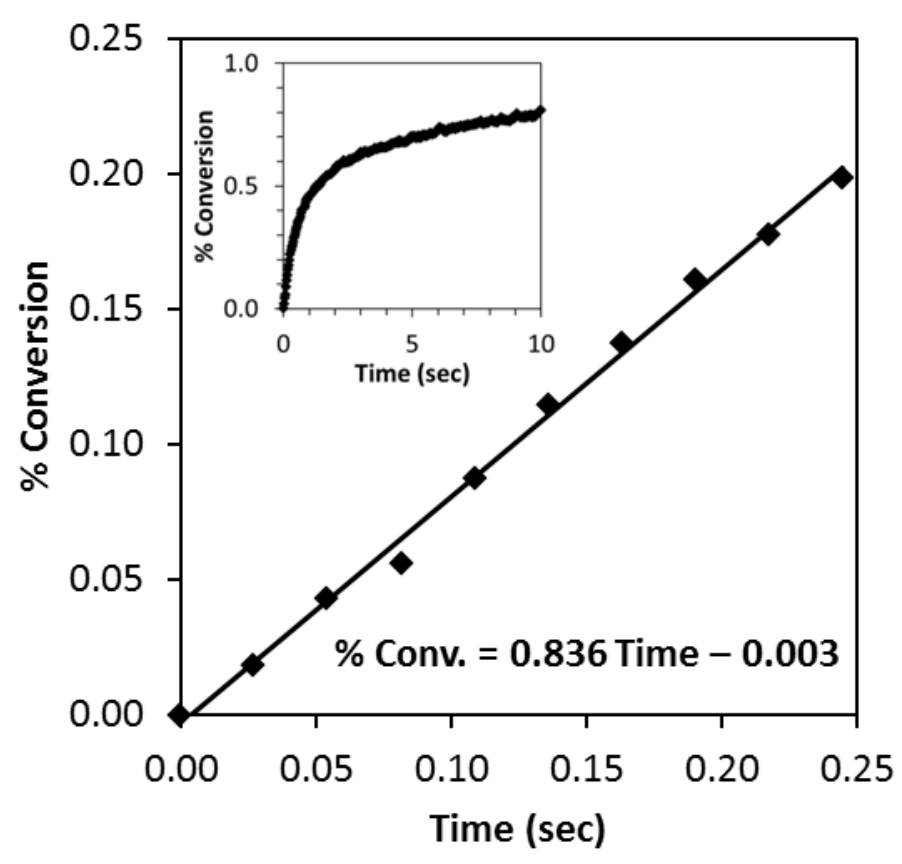

Fig. 3. A representative polymerization kinetic curve of the monomer mix employed in this study. The inset shows the double bond conversion at longer times. leff $_{\text {for }}$ this particular run was $5.27 \mathrm{~mW} / \mathrm{cm}^{2}$ and the gel time was calculated to be $29.0 \mathrm{~ms}$.

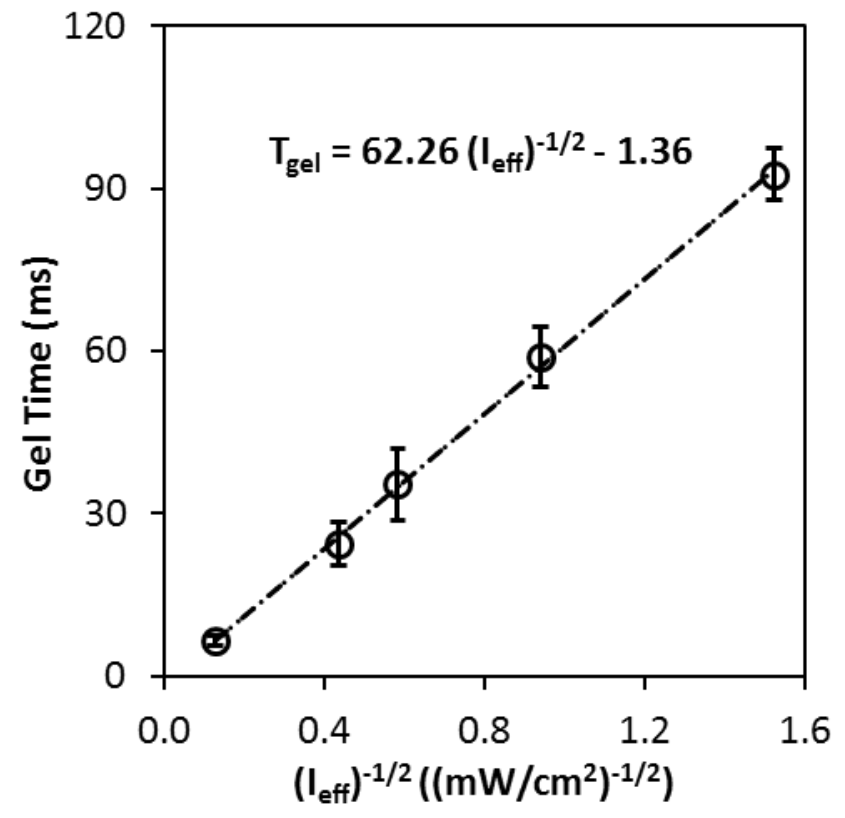


Fig. 4. Gel time of the monomer mix as a function of $\mathrm{l}_{\mathrm{eff}}$ obtained from polymerization kinetics. The dashed line represents the best fit line through all data points. The error bars indicate the standard deviation between four independent measurements at each $\mathrm{l}_{\text {eff. }}$

The gel times were plotted against $\left(\mathrm{l}_{\mathrm{eff}}\right)^{-1 / 2}$ because for a classical free radical polymerization, the polymerization proceeds at a rate proportional to the $-1 / 2$ power of the initiation constant [42], while for a light initiated polymerization such as thiol-ene chemistry, the initiation constant is in turn linearly dependent on the light intensity [43]. The correlation between the gel time and $l_{\text {eff }}$ can thereby be obtained from the RT-FTIR studies as shown by the dashed line in Fig. 4.

Using this correlation, the gel times of monomer mix solutions, exposed to various light intensities in the fiber spinning process, were calculated and presented in Fig. 5 along with the corresponding fiber morphology. As $l_{\text {eff }}$ decreases from 629 to $24 \mathrm{~mW} / \mathrm{cm}^{2}$, the gel time increases exponentially. It was found that in this process, the reaction kinetics need to be sufficiently fast with a gel time less than $2 \mathrm{~ms}$ for defect free fibers to form, as highlighted in the grey shaded region in Fig. 5.

It should be noted that the $l_{\text {eff }}$ in the RT-FTIR studies ranged from 62 to $0.5 \mathrm{~mW} / \mathrm{cm}^{2}$, while the $l_{\text {eff }} s$ in fiber spinning experiments were an order of magnitude higher, ranging from 629 to $21 \mathrm{~mW} / \mathrm{cm}^{2}$. As a result, some of the gel time calculations for the fiber spinning needed to be extrapolated using data presented in Fig. 4. The $l_{\text {eff }}$ in the RT-FTIR studies was intentionally kept low because the polymerization kinetics timescale was approaching the instrument resolution limit. Further increasing the light intensity used in RT-FTIR studies leads 
to a large noise to signal ratio and inconsistent measurements of gel time. In addition, the excellent fit to the linear model with a residual value very close to 1 suggests the validity of data extrapolated to higher light intensity.

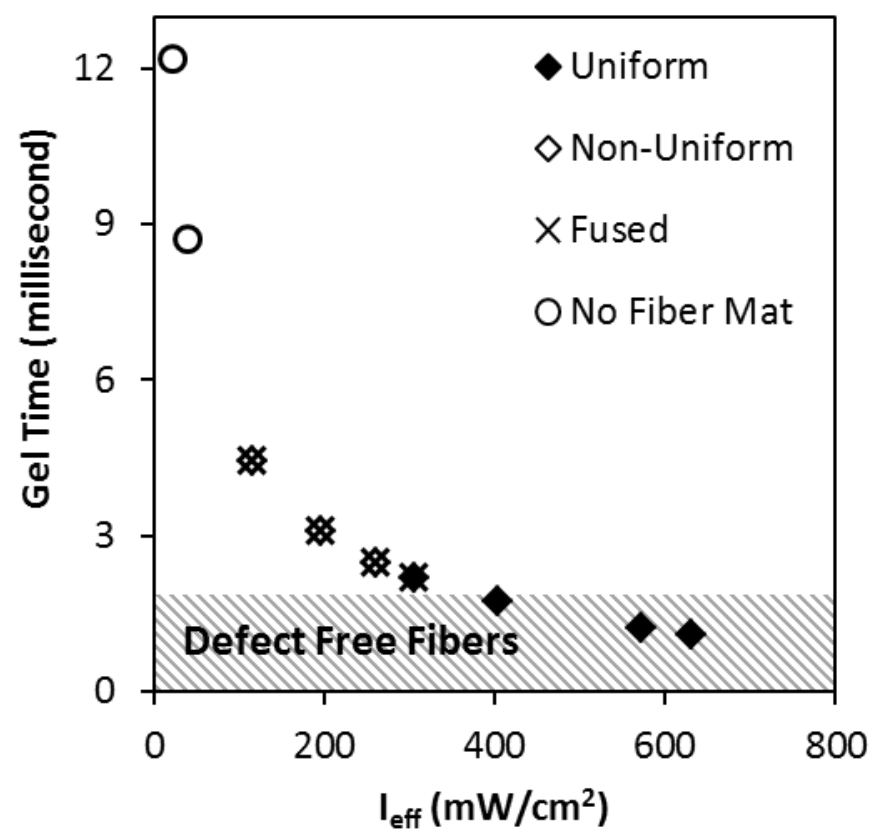

Fig. 5. The gel times of the monomer mix processed at various light intensities. The corresponding fiber morphologies are superimposed on each data point. The shaded region represents the range of gel times of the monomer mix where defect free fiber mats can be produced.

\subsection{Fiber morphology varied by elasticity and characterization of relaxation times}

Next, we discuss the role of elasticity imparted by the high MW PEO on fiber morphology. A series of six monomer mix solutions containing $600,200,100,50,20$, and 0 ppm PEO were prepared and processed at a spin speed of $2500 \mathrm{rpm}$ and $\mathrm{l}_{\text {eff }}$ of $571 \mathrm{~mW} / \mathrm{cm}^{2}$. Such a high $\mathrm{l}_{\text {eff }}$ ensures the rapid curing and solidification of the monomer jets and negates the kinetics 
contribution to morphological evolution in this section. Fig. 6 shows the morphology of the fibers made from these solutions. The fibers transitioned from smooth fiber, to non-uniform fiber, to beads-on-string, and finally to droplets, with decreasing PEO loading. These morphological transitions are similar to the ones caused by reducing $l_{\text {eff }}$ as described in the previous section, but occur due to the decrease in solution elasticity of the fluids. As shown in Fig. 6 (d-f), reducing PEO concentration to a point where the surface tension driven instabilities can no longer be suppressed by elastic forces leads to many defects. It is worth noting that all fiber samples in this section contained no fibers with fused junctions, confirming that the polymerization kinetics are sufficiently fast.
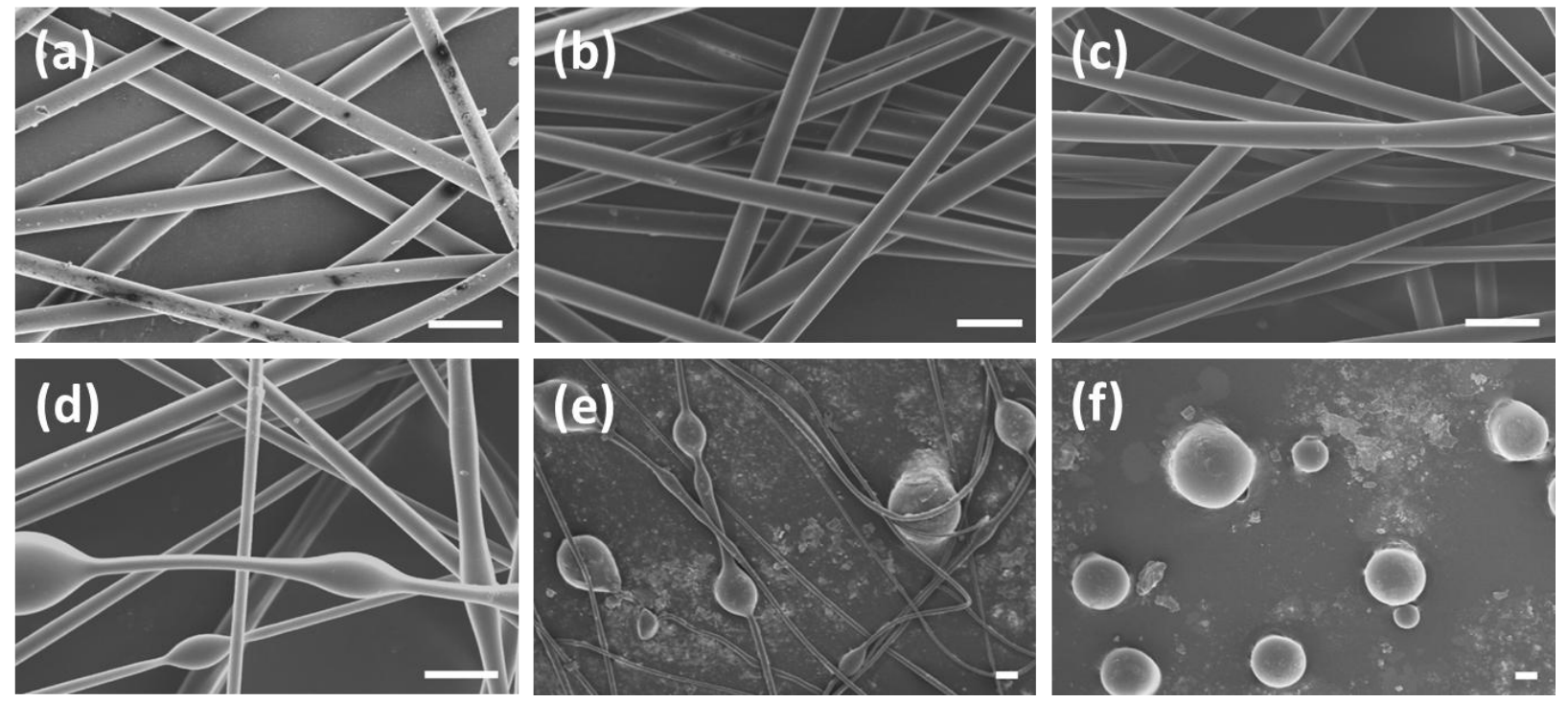

Fig. 6. SEM images of fibers made from monomer mixes containing (a) 600, (b) 200, (c) 100, (d)

50, (e) 20, and (f) 0 ppm PEO, respectively, at a spin speed of $2500 \mathrm{rpm}$, and an leff of

$571 \mathrm{~mW} / \mathrm{cm}^{2}$. The scale bars in this figure correspond to $25 \mu \mathrm{m}$. 
The extensional properties of the monomer mix solutions containing various amounts of PEO can be quantified by their characteristic relaxation times. This timescale can be captured by the time evolution of filament diameter measured by CaBER. As shown in Fig. 7, the decay of the filament diameter of five solutions containing PEO were numerically fit to the elastic fluid model using the following equation [44]:

$$
D(t)=D_{0}\left(\frac{D_{0} G}{4 \sigma}\right)^{\frac{1}{3}} e^{-\frac{t}{\lambda_{c}}}
$$

where $D(t)$ is the time dependent filament diameter measured at the midpoint of the filament by the CaBER micrometer, $D_{0}$ is the initial diameter of the filament, $G$ is the elastic modulus, $\sigma$ the surface tension, and $\lambda_{c}$ is the characteristic relaxation time of the monomer mix solution. The characteristic relaxation times of the solutions were extracted by fitting the data to this model and are reported in Fig. 8. The error bars in Fig. 8 represent the standard deviations from five CaBER measurements for each solution.

On the other hand, the decay of the filament diameter of the solution containing no PEO was best described by the Newtonian fluid model using the following equation $[45,46]$ :

$$
D(t)=0.1418 \frac{\sigma}{\eta_{s}}\left(t_{c}-t\right) \quad(\text { Eq. 3) }
$$

where $t_{c}$ is the critical time to break up, and $\eta_{s}$ is the viscosity of the solvent, which is the viscosity of the entire monomer mix containing no PEO, measured to be 0.24 Pa-s by shear rheometry (TA instruments AR-2000 EX). The excellent fit to the Newtonian fluid model is expected since the solution was inelastic without PEO. 


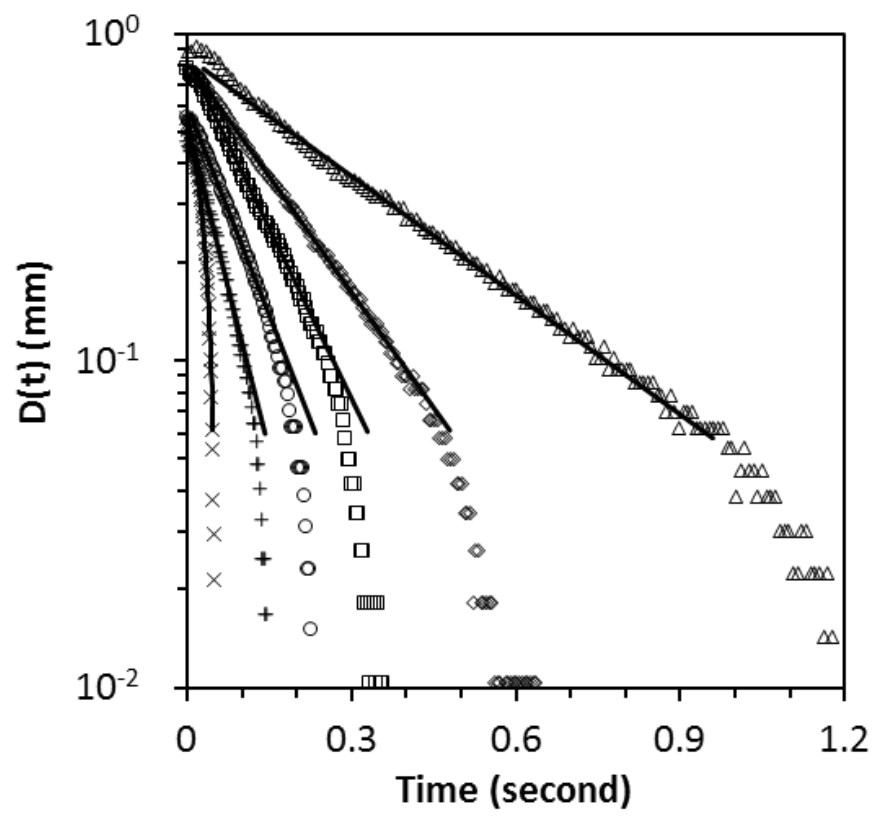

Fig. 7. Evolution of the midpoint filament diameter $D(t)$ during filament thinning of monomer mix solutions containing $600 \mathrm{ppm}(\triangle), 200 \mathrm{ppm}(\diamond), 100 \mathrm{ppm}(\square), 50 \mathrm{ppm}(0), 20 \mathrm{ppm}(+)$, and 0 ppm ( $\times$ ) PEO, respectively. Solid lines are best fit lines to either the elastic fluid model (600 ppm to $20 \mathrm{ppm}$ PEO) or the Newtonian fluid model (0 ppm PEO).

In Fig. 8, the characteristic fluid relaxation times of the PEO containing solutions are plotted as a function of the PEO concentrations by weight. The dashed line represents the least squares power law fit to the data with an exponent of 0.486 . Previously, the concentration dependence of relaxation times of unentangled semi-dilute polymer solutions has been established and empirically determined to follow a power law dependence. In this regime, the power law scaling exponent, equal to $(2-3 v) /(3 v-1)$, is equal to 1 for a $\theta$-solvent and around 0.31 for a good solvent. The symbol v represents the excluded volume scaling parameter, which indicates the solvent quality for a polymer and spans from 0.5 for a $\theta$-solvent to 0.588 for a good solvent [47]. This scaling law has been verified by several experiments with different semi- 
dilute polymer-solvent systems [48-50]. The good fit of our data to the power law equation with an exponent between 0.31 and 1 confirms that the series of solutions with various PEO concentrations falls in the dilute unentangled polymer solution regime. In addition, the scaling exponent of 0.486 from the fit in this study corresponds to $v=0.558$, which indicates that the monomer mix solution is a fair solvent for PEO, but not as good as that of water, a commonly used solvent for PEO [34, 51, 52]. Furthermore, the good fit in Fig. 8 demonstrates that, although the monomer molecules are relatively large in terms of molecular weight, they essentially behave like other conventional small molecule organic solvents for the dissolution of PEO.

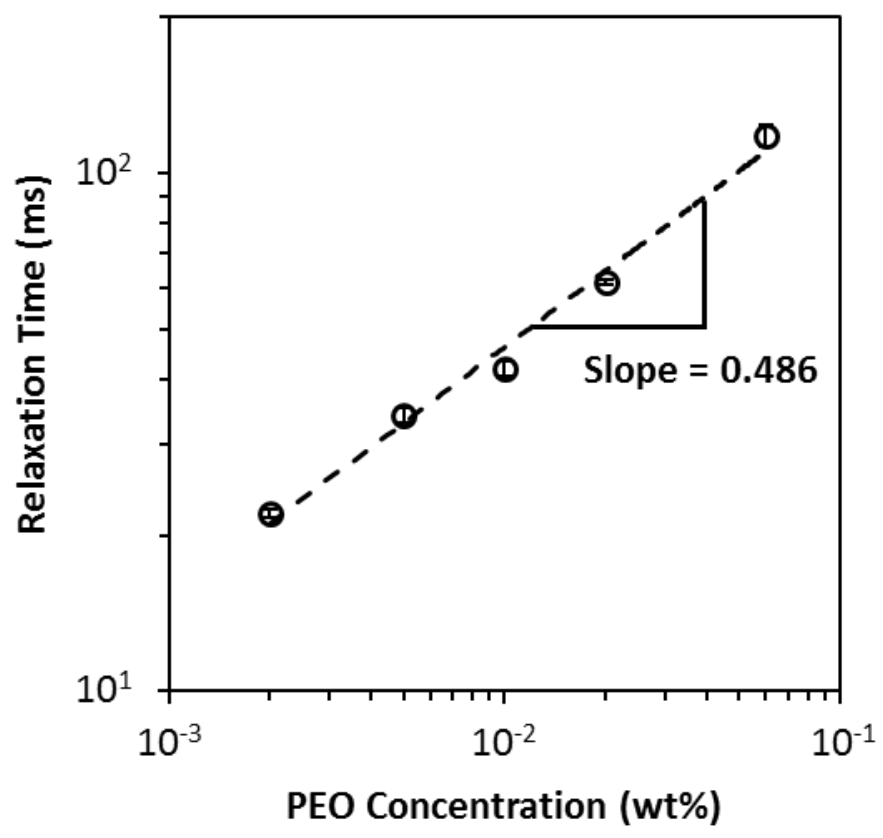

Fig. 8. Relaxation time of monomer mix solutions as a function of the concentration of PEO. The dashed line represents a least squares power law fit to the data. The error bars are standard deviations of five independent CaBER measurements. 
In addition to fluid relaxation times, the apparent extensional viscosity $\left(\eta_{\text {app }}\right)$, of the monomer mix solution as a function of Hencky strain $(\varepsilon)$, can be calculated using the following equations [44]:

$$
\begin{gathered}
\varepsilon=\ln \left(\frac{D(t)}{D_{0}}\right) \\
\eta_{a p p}(\varepsilon)=-\frac{\sigma}{d D(t) / d t}
\end{gathered}
$$

In CaBER experiments, filament thinning is solely driven by surface tension, while in centrifugal spinning, it is driven by both inertia and surface tension. Despite the differences, this analysis still captures the viscoelastic response for an exponentially thinning filament. The results are presented in Fig. 9. Expectedly, the solution containing no PEO behaves like a Newtonian fluid, and the apparent extensional viscosity is independent of Hencky strain, whereas the solutions containing PEO show the strain hardening effect, which becomes more prominent with increasing PEO concentration.

$\eta_{a p p}$ of the monomer mix solution containing 200 ppm PEO (the typical PEO loading for the monomer mix) increases by almost two orders of magnitude over the range of Hencky strains accessible in CaBER studies, showing that adding a trace amount of high MW PEO can significantly enhance the extensional behavior of the solution. In contrast, the shear viscosities of the solutions containing $0 \mathrm{ppm}$ and $600 \mathrm{ppm}$ PEO were measured by a parallel plate rheometer to be 0.24 and $0.33 \mathrm{~Pa}-\mathrm{s}$, respectively (Fig. S4 in Supporting Information). The dramatic change in $\eta_{a p p}$ combined with the narrow range of the shear viscosity indicates that these solutions are typical Boger fluids, whose extensional properties can be tuned independently of their shear properties [53]. A Boger fluid is commonly a dilute polymer 
solution with a viscous solvent [53], which in this study is a dilute PEO solution with a viscous thiol-ene monomer mix.

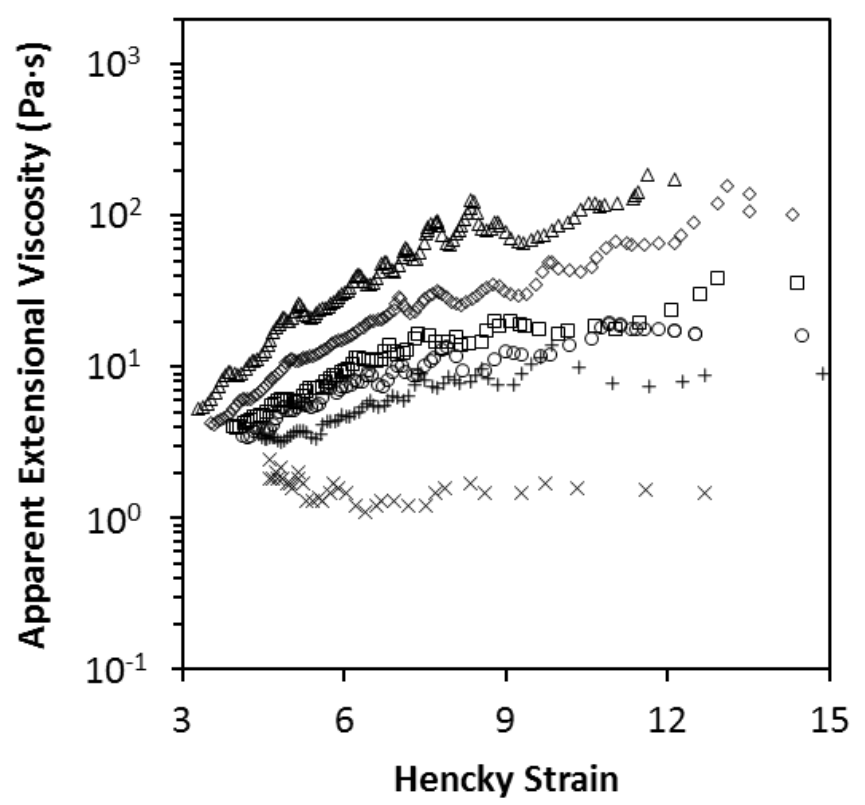

Fig. 9. Apparent extensional viscosities of the monomer mix solutions containing $600 \mathrm{ppm}(\triangle)$,

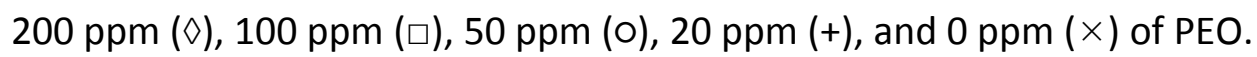

The evolution of fiber morphology from uniform fiber, non-uniform fiber, beads-on-string, and finally droplets depends on the balance between the viscous, elastic, inertial stresses, thinning due to centrifugal force and the capillary pressure inside the jet. In order to evaluate quantitatively the relative importance of each force, we introduce three dimensionless parameters: the Deborah number (De), Weissenberg number (Wi), and Ohnesorge number (Oh). De measures the ratio of the characteristic timescale of fluid relaxation to the timescale of Rayleigh inertio-capillary breakup, and is defined as $D e=\lambda_{c} / \sqrt{\rho R^{3} / \sigma}[45,52]$, where $\mathrm{R}$ is the radius of the initial fluid jet $(0.105 \mathrm{~mm})$. Using the relaxation times obtained from the CaBER 
experiments, the calculated De numbers for solutions containing 20, 50, 100, 200, 600 ppm PEO are $3.8,6.0,7.3,10.8$, and 20.6, respectively. For our system and process, uniform fiber formation requires the De to be well above unity ( $D e>7.3$ ), where fluid relaxation time is much greater than the Rayleigh instability timescale, and the onset of capillary breakup is completely suppressed by the elastic stresses. When Rayleigh instabilities are not completely suppressed (De $<7.3$ ), significant beading in monomer jets is observed, leading to a beads-on-string morphology. If the elastic response is absent altogether, as in the case of the monomer mix containing no elasticity modifier, Rayleigh instabilities develop and the monomer jets readily dissipate into droplets upon exiting the spinneret. The fiber morphology developed with reducing De number is very similar to a study reported by Yu and coworkers on electrospinning PEO in water/PEG solutions [34].

On the other hand, $\mathrm{Wi}$ is defined as the ratio of the characteristic timescales of fluid relaxation to fiber thinning due to centrifugal force, and can be expressed as $W i=\lambda_{c} \Omega / \sqrt{R / a}$, where $\Omega$ is the spin speed and $a$ is the distance between the orifice and the spinning axis [54]. Wi numbers for monomer mix solutions containing $20,50,100,200,600$ ppm PEO are 30.3, $46.9,57.7,84.8$, and 162.4 , respectively. The transition from uniform fibers to beads-on-string or tobead formation occurs when Wi number falls below 46.9, again demonstrating the significance of elastic stresses for fiber formation. This correlation agrees well with a recent mechanistic study on solution state centrifugal spinning by Ren et al, where it was found for a variety of polymer-solvent systems and various processing conditions that fibers were formed when Wi numbers exceeded 28, while beads-on-string and beads were formed if Wi numbers fell below 28 [54]. 
These interesting similarities of fiber morphology development as a function of De and Wi suggest that, despite the drastic differences in mechanisms of fluid jet solidification, the underlying fiber drawing forces and fiber trajectory, the elastic forces and conditions required to form stable fluid jet and uniform fibers in this reactive fiber spinning method are comparable to that of a non-reactive fiber spinning method, provided sufficiently rapid curing kinetics.

In contrast, the Ohnesorge number relates viscous forces to inertial and surface tension forces, and is defined as $O h=\eta / \sqrt{\rho \sigma R}[45,52]$, where $\eta$ is the viscosity of the monomer mix solution. The Ohnesorge number is around 4 for all solutions, because the addition of PEO does not significantly influence the viscosity, density, and surface tension of the monomer mix solutions. An Oh number greater than unity also implies that viscous stress is enough to stabilize the filament, again confirming that the morphological changes mainly arise as a result of changes in solution elastic properties.

\subsection{Fiber morphology evolved with spin speed and estimation of flight time}

In addition to gel time and fluid relaxation time, the fiber jet flight time is another important time scale in this process because it dictates the available time for light exposure and photopolymerization of the monomer jet before it hits the collector. The monomer mix solution that makes good fibers at $2500 \mathrm{rpm}$ (as shown in Fig. 2b) was also processed at $3500 \mathrm{rpm}$ and $4500 \mathrm{rpm}$ using the same illumination condition, as shown in Fig. 10. Fibers processed at 3500 rpm were well cured (Fig. 10a) while fibers spun at $4500 \mathrm{rpm}$ were fused at their junctions (Fig. 10b). At $4500 \mathrm{rpm}$, the fibers did not have enough time to cure completely before depositing onto other fibers on the collector. 

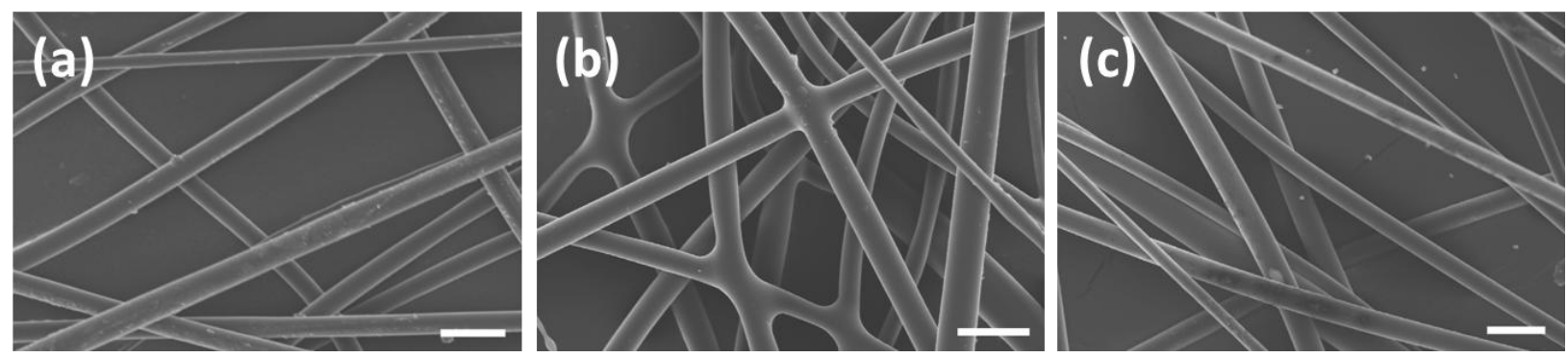

Fig. 10. SEM images of fibers at a spin speed of (a) $3500 \mathrm{rpm}$ and (b, c) $4500 \mathrm{rpm}$. The monomer mix contains 3 wt\% Irgacure 2100 in $(a, b)$ and 5 wt\% Irgacure 2100 in (c). The PEO loading is $200 \mathrm{ppm}$ and the $\mathrm{I}_{\text {eff }}$ is $571 \mathrm{~mW} / \mathrm{cm}^{2}$ for all three runs. The scale bars in this figure correspond to $25 \mu \mathrm{m}$.

The flight time can be defined as the fiber flight distance divided by the fiber velocity. In centrifugal Forcespinning, the fluid jet follows a spiral trajectory outward from the spinneret tip to the vertical collector bars, which depends on spin speed, solution viscosity and elasticity, ratio of length to diameter of the orifice and other process variables [55-57]. In addition, the fiber velocity is not constant as the fluid jet attenuates in diameter [55]. Both parameters are difficult to assess accurately by either empirical measurement or theoretical calculation. In this study, the flight time is estimated by using the diameter of the beam spot on the circular collector $(10 \mathrm{~cm})$ as the flight distance and the linear velocity of the tip of the spinneret as the fiber velocity. Although the fiber does not travel in a linear fashion through the center of the illuminated region, this is the most straightforward estimate for this reactive fiber spinning process. The linear velocity of the spinneret tip can also be viewed as the initial velocity of the fiber as it exits the spinneret. For a rotation radius of $11.2 \mathrm{~cm}$, the initial linear fiber velocities are $29.8,41.8,53.7 \mathrm{~m} / \mathrm{s}$ at rotational spin speeds of 2500,3500 , and 4500 , respectively. The 
respective flight times of fiber jets processed at 2500, 3500, and $4500 \mathrm{rpm}$ are $3.4,2.4$, and 1.9 ms. With all other conditions and material properties being the same, a minimum of $2.4 \mathrm{~ms}$ flight time was required to produce defect free fibers.

However, flight time is meaningful only when it is placed in context with other timescales. For example, avoiding the formation of fused fibers can be achieved in several ways, such as increasing flight time by spinning at lower spin speeds as shown in Fig. 10 (a) and decreasing gel time by increasing reaction kinetics. Fig. 10 (c) is an SEM image of fibers made at the exact same spinning and lighting condition as Fig. 10 (b), but with a monomer mix that contained 5 wt\% of photoinitiator instead of $3 \mathrm{wt} \%$. The higher loading of photoinitiator increases the curing speed, allowing the fiber jet to cure completely before deposition. This again exemplifies the complexity and the intertwined variables of this simultaneous fiber spinning and photopolymerization process.

\subsection{Operating diagram describing the interactions of the three timescales}

The process conditions, the corresponding timescales, and fiber morphologies of the three fiber spinning test conducted in this study are summarized in Table 1. Also presented are the ratio of fluid relaxation time $\left(T_{\text {relax }}\right)$ to gel time $\left(T_{\text {gel }}\right)$, and the ratio of flight time $\left(T_{\text {flight }}\right)$ to $T_{\text {gel }}$. An operating diagram is constructed by plotting the ratios of timescales along with the fiber morphologies as shown in Fig. 11.

Table 1. A summary of fiber spinning conditions, related time scales, and fiber morphology. The first row in each data set indicates the variable of interest and the other parameter values that were held constant in the experimental runs below the first row. 


\begin{tabular}{|c|c|c|c|c|c|c|c|c|c|}
\hline Variable & $\begin{array}{c}\mathrm{I}_{\text {eff }} \\
\left(\mathrm{mW} / \mathrm{cm}^{2}\right)\end{array}$ & $\begin{array}{c}\mathrm{C}_{\text {PEO }} \\
(\mathrm{ppm})\end{array}$ & $\begin{array}{c}\text { Spin Speed } \\
(\mathrm{rpm})\end{array}$ & $\begin{array}{c}T_{\text {gel }} \\
(\mathrm{ms})\end{array}$ & $\begin{array}{c}\mathrm{T}_{\text {relaxation }} \\
\text { (ms) }\end{array}$ & $\begin{array}{l}T_{\text {flight }} \\
\text { (ms) }\end{array}$ & $\mathrm{T}_{\text {relaxation }} / \mathrm{T}_{\text {gel }}$ & $\mathrm{T}_{\text {flight }} / \mathrm{T}_{\text {gel }}$ & Fiber Morphology \\
\hline \multirow[t]{10}{*}{$I_{\text {eff }}$} & & 200 & 2500 & & 61.7 & 3.4 & & & \\
\hline & 629 & & & 1.1 & & & 54.9 & 3.0 & Uniform, cured \\
\hline & 571 & & & 1.2 & & & 49.5 & 2.7 & Uniform, cured \\
\hline & 403 & & & 1.7 & & & 35.4 & 2.0 & Uniform, cured \\
\hline & 305 & & & 2.2 & & & 28.0 & 1.5 & Uniform, fused \\
\hline & 259 & & & 2.5 & & & 24.6 & 1.4 & Wavy, fused \\
\hline & 195 & & & 3.1 & & & 19.9 & 1.1 & Wavy, fused \\
\hline & 115 & & & 4.4 & & & 13.9 & 0.8 & Wavy, fused \\
\hline & 38 & & & 8.7 & & & 7.1 & 0.4 & Beads-on-string, fused \\
\hline & 21 & & & 12.2 & & & 5.1 & 0.3 & Droplet \\
\hline \multirow[t]{7}{*}{$\mathrm{C}_{\text {PEO }}$} & 571 & & 2500 & 1.2 & & 3.4 & & & \\
\hline & & 600 & & & 118.1 & & 94.8 & 2.7 & Uniform, cured \\
\hline & & 200 & & & 61.7 & & 49.5 & 2.7 & Uniform, cured \\
\hline & & 100 & & & 41.9 & & 33.7 & 2.7 & Uniform, cured \\
\hline & & 50 & & & 34.1 & & 27.4 & 2.7 & Wavy, cured \\
\hline & & 20 & & & 22.0 & & 17.7 & 2.7 & Beads-on-string, cured \\
\hline & & 0 & & & NA & & NA & 2.7 & Droplet \\
\hline Spin & 571 & 200 & & 1.2 & 61.7 & & & & \\
\hline \multirow[t]{3}{*}{ Speed } & & & 2500 & & & 3.4 & 49.5 & 2.7 & Uniform, cured \\
\hline & & & 3500 & & & 2.4 & 49.5 & 1.9 & Uniform, cured \\
\hline & & & 4500 & & & 1.9 & 49.5 & 1.5 & Uniform, fused \\
\hline
\end{tabular}
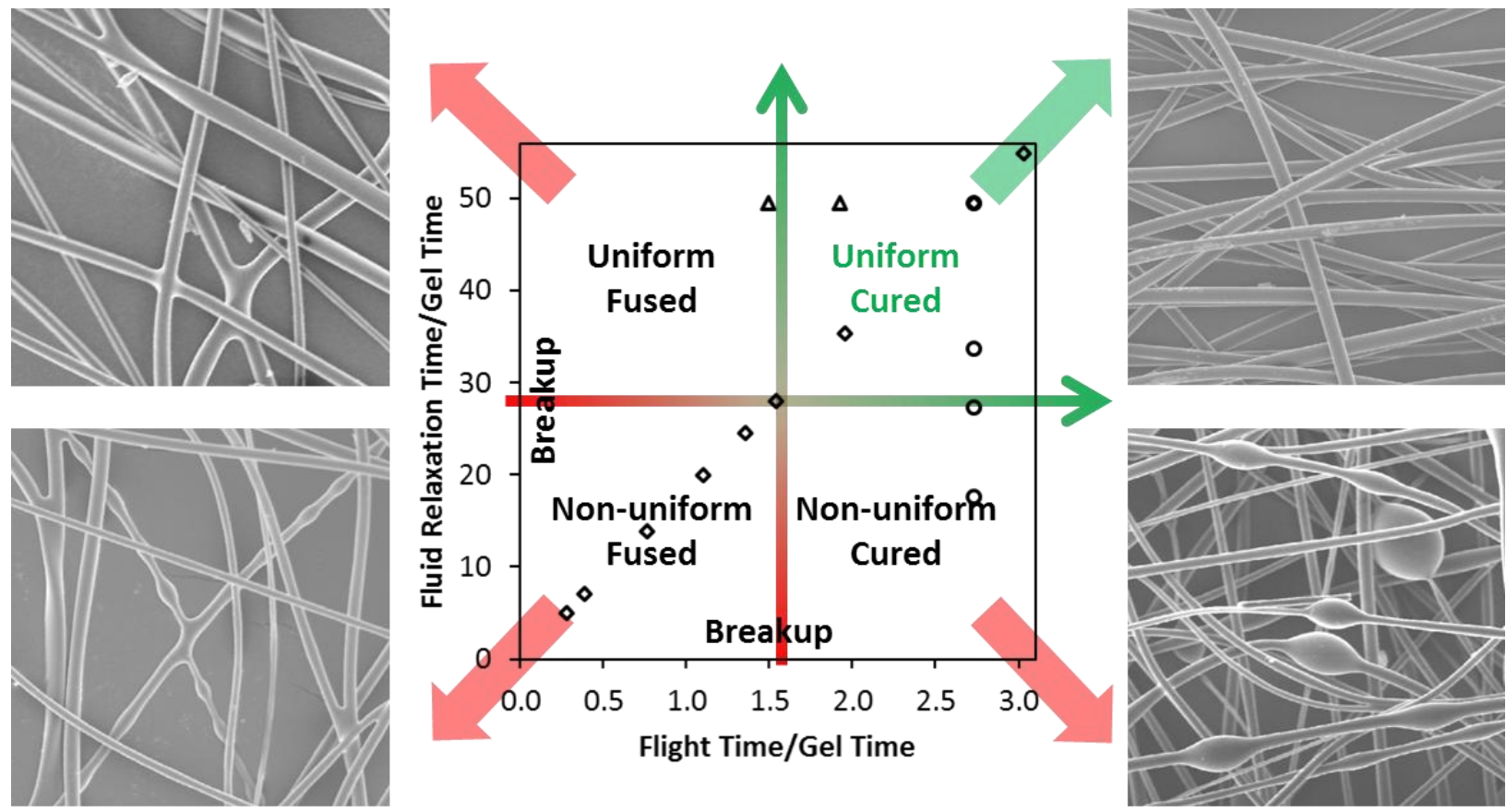

Fig. 11. Operating diagram relating fluid relaxation time, gel time, and flight time in the reactive centrifugal fiber spinning process. $\diamond, 0, \triangle$ represent the fiber sample series made by varying $l_{\text {eff, }}$ $C_{P E O}$, and spin speed, respectively. 
Despite the complexity of this multivariable process, the fiber morphology evolves as a result of the competition between three phenomena: the solidification by thiol-ene photopolymerization, the surface tension driven instability growth which can be suppressed by solution elasticity, and the orifice-to-collector flight time, characterized by $T_{\text {gel, }}, T_{\text {relax }}$, and $T_{\text {flight, }}$ respectively. In Fig. 11, the horizontal arrow indicates the threshold value for the formation of uniform fiber, whereas the vertical arrow indicates the threshold value for the formation of well cured fiber. Defect free fibers are formed when gel time is sufficiently small compared to both the fluid relaxation time and the flight time. When a mismatch of timescales occurs such that either one or both ratios fall below the threshold value, fiber defects such as uncured fibers, non-uniform fibers, or a combination of the two develop. When timescales become significantly mismatched, fiber jets readily dissipate into droplets.

\section{Conclusion}

In this article, we have demonstrated the fabrication of fibers by simultaneous thiol-ene photopolymerization and centrifugal Forcespinning an optimized formulation containing multifunctional thiol and ene monomers, photoinitiator, high MW PEO as a elasticity modifier, and ethyl acetate as a diluent. Fundamental understanding on conditions that lead to a variety of fiber morphologies, including droplets, beads-on-string, uniform fibers, fused fibers, and well cured fibers was developed by systematically varying three carefully chosen parameters: light intensity, PEO concentration, and spin speed, which independently affect the reaction kinetics, solution viscoelasticity, and orifice-to-collector flight time, respectively. To quantify these 
transitions further, the relevant timescales, namely the gel time, the characteristic relaxation time, and the fiber flight time, at each fiber spinning condition were measured, calculated and compared.

An operating diagram was constructed by comparing ratios of the three timescale measurements at each fiber spinning condition, which explicitly illustrates that the fiber morphology is determined not only by the individual parameters of the process, but also by the interplay between them. Although there are numerous combinations of these parameters, the ultimate fiber morphology will always be governed by the relative speeds of solidification by photopolymerization, surface tension driven instability growth, and fiber deposition. Our qualitative understanding of the fundamental physics and quantitative operating diagram for controlling fiber formation and morphology can serve as a predictive guideline for the implementation of photopolymerization into other fiber spinning processes and the fabrication of fibers with designed morphology and function.

\section{Acknowledgements}

We gratefully acknowledge partial financial support from the Welch Foundation (grant \#F-1709) and the National Science Foundation through the CLiPs NSF-STC, DMR-0423914. CJE gratefully acknowledges partial financial support from the DuPont Young Professor Award and 3M NonTenured Faculty Award. We also gratefully acknowledge help from Andrew Worthen and Prof. Keith Johnston for their help in taking surface tension measurements.

\section{References}


1. Wang N, Wang X, Ding B, Yu J, and Sun G. Journal of Materials Chemistry 2012;22(4):1445-1452.

2. Yun KM, Suryamas AB, Iskandar F, Bao L, Niinuma H, and Okuyama K. Separation and purification technology 2010;75(3):340-345.

3. Ma M, Gupta M, Li Z, Zhai L, Gleason KK, Cohen RE, Rubner MF, and Rutledge GC. Advanced Materials 2007;19(2):255-259.

4. Ma M, Mao Y, Gupta M, Gleason KK, and Rutledge GC. Macromolecules 2005;38(23):9742-9748.

5. Wang Z, Macosko CW, and Bates FS. ACS applied materials \& interfaces 2014;6(14):11640-11648.

6. Radhakrishnan L, Reboul J, Furukawa S, Srinivasu P, Kitagawa S, and Yamauchi Y. Chemistry of Materials 2011;23(5):1225-1231.

7. Badami AS, Kreke MR, Thompson MS, Riffle JS, and Goldstein AS. Biomaterials 2006;27(4):596606.

8. Buttafoco L, Kolkman N, Engbers-Buijtenhuijs P, Poot A, Dijkstra P, Vermes I, and Feijen J. Biomaterials 2006;27(5):724-734.

9. Moroni L, Licht R, de Boer J, de Wijn JR, and van Blitterswijk CA. Biomaterials 2006;27(28):49114922.

10. Oliveira JE, Moraes EA, Costa RG, Afonso AS, Mattoso LH, Orts WJ, and Medeiros ES. Journal of Applied Polymer Science 2011;122(5):3396-3405.

11. Zhmayev E, Cho D, and Joo YL. Polymer 2010;51(18):4140-4144.

12. Cho D, Zhmayev E, and Joo YL. Polymer 2011;52(20):4600-4609.

13. Sarkar K, Gomez C, Zambrano S, Ramirez M, de Hoyos E, Vasquez H, and Lozano K. Materials Today 2010;13(11):12-14.

14. Fang Y, Herbert M, Schiraldi DA, and Ellison CJ. Journal of Materials Science 2014;49(24):82528260.

15. Hammami MA, Krifa M, and Harzallah O. The Journal of The Textile Institute 2014;105(6):637647.

16. Shanmuganathan K, Fang Y, Chou DY, Sparks S, Hibbert J, and Ellison CJ. ACS Macro Letters 2012;1(8):960-964.

17. Weng B, Xu F, Salinas A, and Lozano K. Carbon 2014;75:217-226.

18. Tate BD and Shambaugh RL. Industrial \& engineering chemistry research 2004;43(17):5405-5410.

19. Shanmuganathan K, Sankhagowit RK, lyer P, and Ellison CJ. Chemistry of Materials 2011;23(21):4726-4732.

20. Janes DW, Shanmuganathan K, Chou DY, and Ellison CJ. ACS Macro Letters 2012;1(9):1138-1142.

21. Shanmuganathan K, Elliot SM, Lane AP, and Ellison CJ. ACS applied materials \& interfaces 2014;6(16):14259-14265.

22. Boyd DA, Shields AR, Naciri J, and Ligler FS. ACS applied materials \& interfaces 2012;5(1):114119.

23. Yang $\mathrm{H}$, Zhang $\mathrm{Q}$, Lin B, Fu G, Zhang X, and Guo L. Journal of Polymer Science Part A: Polymer Chemistry 2012;50(20):4182-4190.

24. Kim SH, Kim S-H, Nair S, and Moore E. Macromolecules 2005;38(9):3719-3723.

25. Ji Y, Ghosh K, Li B, Sokolov JC, Clark RA, and Rafailovich MH. Macromolecular bioscience 2006;6(10):811-817.

26. Jarusuwannapoom T, Hongrojjanawiwat W, Jitjaicham S, Wannatong L, Nithitanakul M, Pattamaprom C, Koombhongse P, Rangkupan R, and Supaphol P. European Polymer Journal 2005;41(3):409-421.

27. Uyar T and Besenbacher F. Polymer 2008;49(24):5336-5343.

28. Megelski S, Stephens JS, Chase DB, and Rabolt JF. Macromolecules 2002;35(22):8456-8466.

29. Gupta P, Elkins C, Long TE, and Wilkes GL. Polymer 2005;46(13):4799-4810.

30. Shenoy SL, Bates WD, Frisch HL, and Wnek GE. Polymer 2005;46(10):3372-3384. 
31. McKee MG, Wilkes GL, Colby RH, and Long TE. Macromolecules 2004;37(5):1760-1767.

32. Nie H, He A, Zheng J, Xu S, Li J, and Han CC. Biomacromolecules 2008;9(5):1362-1365.

33. Min B-M, Lee SW, Lim JN, You Y, Lee TS, Kang PH, and Park WH. Polymer 2004;45(21):7137-7142.

34. Yu JH, Fridrikh SV, and Rutledge GC. Polymer 2006;47(13):4789-4797.

35. Palangetic L, Reddy NK, Srinivasan S, Cohen RE, McKinley GH, and Clasen C. Polymer 2014;55(19):4920-4931.

36. Crowley MM, Zhang F, Koleng JJ, and McGinity JW. Biomaterials 2002;23(21):4241-4248.

37. Adkins SS, Chen X, Nguyen QP, Sanders AW, and Johnston KP. Journal of colloid and interface science 2010;346(2):455-463.

38. Reddy SK, Okay O, and Bowman CN. Macromolecules 2006;39(25):8832-8843.

39. Cramer NB and Bowman CN. Journal of Polymer Science Part A: Polymer Chemistry 2001;39(19):3311-3319.

40. Reddy SK, Cramer NB, and Bowman CN. Macromolecules 2006;39(10):3681-3687.

41. Cramer NB, Davies T, O'Brien AK, and Bowman CN. Macromolecules 2003;36(12):4631-4636.

42. Rodriguez F, Cohen C, Ober C, and Archer LA. Principles of polymer systems: Taylor \& Francis Washington, DC, 1996.

43. Anseth KS, Bowman CN, and Peppas NA. Journal of Polymer Science Part A: Polymer Chemistry 1994;32(1):139-147.

44. Regev O, Vandebril S, Zussman E, and Clasen C. Polymer 2010;51(12):2611-2620.

45. Campo-Deano L and Clasen C. Journal of Non-Newtonian Fluid Mechanics 2010;165(23):16881699.

46. McKinley GH and Tripathi A. Journal of Rheology (1978-present) 2000;44(3):653-670.

47. Rubinstein M and Colby R. Polymers Physics: Oxford, 2003.

48. Patel SS and Takahashi KM. Macromolecules 1992;25(17):4382-4391.

49. Liu Y, Jun Y, and Steinberg V. Journal of Rheology (1978-present) 2009;53(5):1069-1085.

50. Hur JS, Shaqfeh ES, Babcock HP, Smith DE, and Chu S. Journal of Rheology (1978-present) 2001;45(2):421-450.

51. Tirtaatmadja V, McKinley GH, and Cooper-White JJ. Physics of Fluids (1994-present) 2006;18(4):043101.

52. Oliveira MS, Yeh R, and McKinley GH. Journal of non-newtonian fluid mechanics 2006;137(1):137-148.

53. James DF. Annual Review of Fluid Mechanics 2009;41:129-142.

54. Ren L, Ozisik R, Kotha SP, and Underhill PT. Macromolecules 2015.

55. Taghavi S and Larson R. Physical Review E 2014;89(2):023011.

56. Xu H, Chen H, Li X, Liu C, and Yang B. Journal of Polymer Science Part B: Polymer Physics 2014.

57. Padron S, Fuentes A, Caruntu D, and Lozano K. Journal of Applied Physics 2013;113(2):024318. 

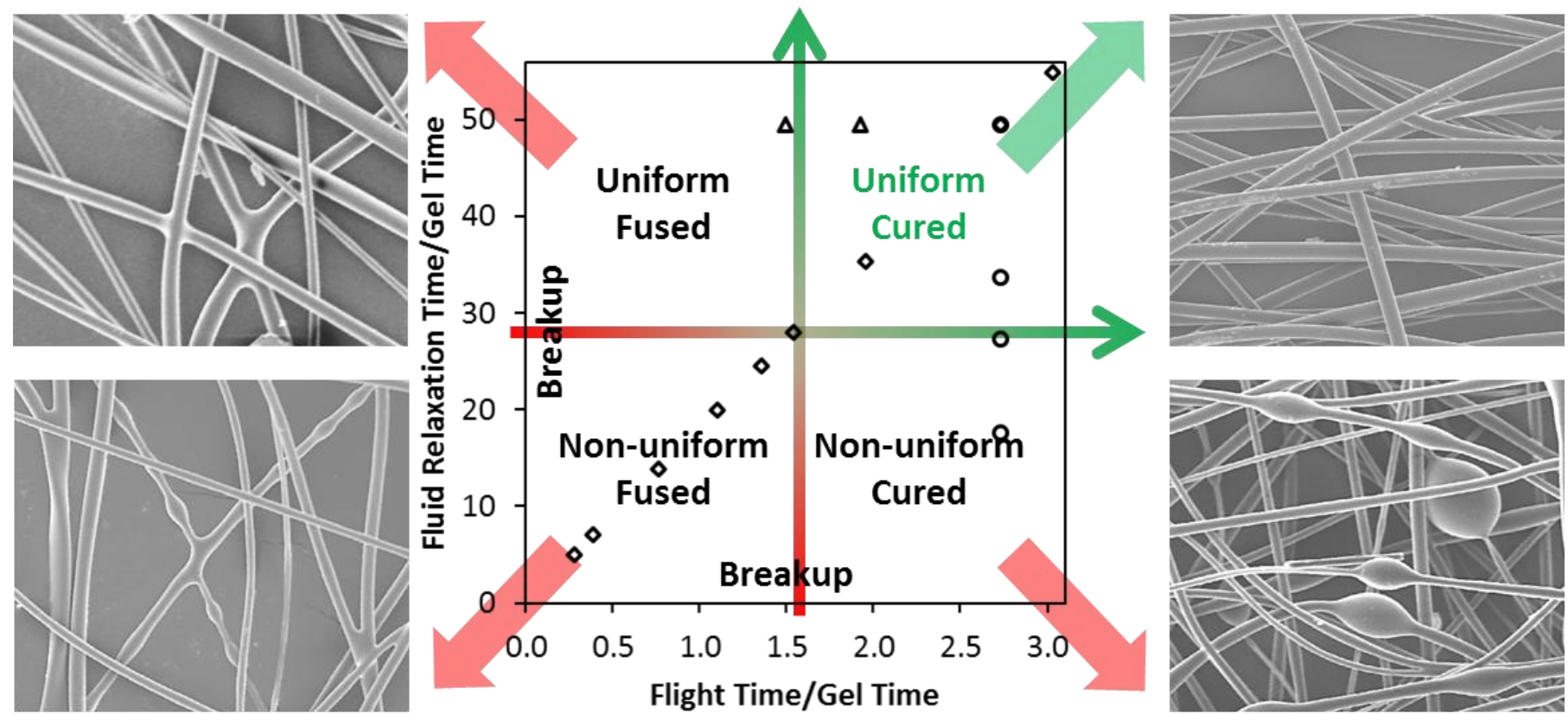

Flight Time/Gel Time

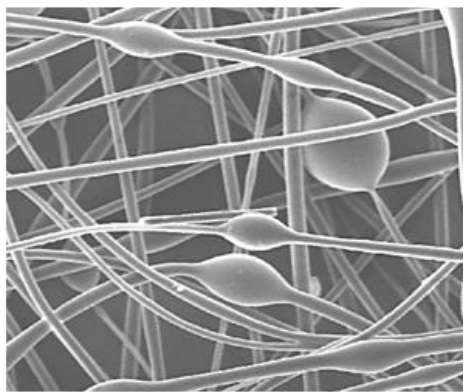

